
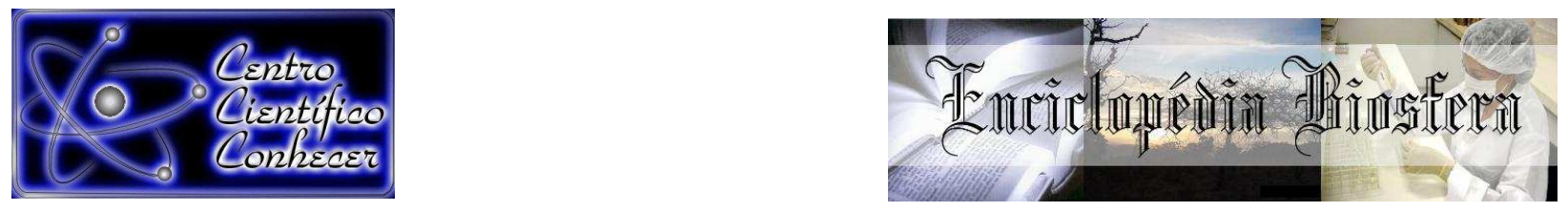

\title{
CASOS DE MALÁRIA REGISTRADOS NO MUNICÍPIO DE CÂNDIDO MENDES, MARANHÃO, NO PERÍODO DE 2009-2014
}

\author{
Yuri Freitas da Silva ${ }^{1}$, Ana Lúcia Nunes Gutjahr², Carlos Elias de Souza \\ Braga $^{3}$, João Raimundo Alves Marques ${ }^{4}$ \\ ${ }^{1}$ Licenciado em Ciências Naturais, Universidade do Estado do Pará, Belém-PA \\ (yuri.freitas@hotmail.com),Brasil \\ 2Professora Doutora, Universidade do Estado do Pará, Departamento de Ciências \\ Naturais, Belém-PA, Brasil \\ ${ }^{3}$ Professor Doutor, Universidade do Estado do Pará, do Departamento de Ciências \\ Naturais, Paragominas-PA, Brasil \\ ${ }^{4}$ Mestrando em Ciências Ambientais, Universidade do Estado do Pará, Belém-PA, \\ Brasil
}

Recebido em: 08/04/2017 - Aprovado em: 10/06/2017 - Publicado em: 20/06/2017 DOI: 10.18677/EnciBio_2017A127

\begin{abstract}
RESUMO
Este estudo objetivou descrever os aspectos epidemiológicos da malária no município de Cândido Mendes, Maranhão, baseado nos casos notificados no período de 2009 a 2014. Para isso, realizou-se um levantamento dos dados do Sistema Único de Saúde (SUS) e Sistema de Informação de Vigilância Epidemiológica para malária (SIVEP-MALÁRIA), de janeiro de 2009 a dezembro de 2014. Os resultados evidenciam a presença da malária no município, com maior incidência em 2009 (346 casos) e redução gradual de casos nos anos seguintes, exceto no ano de 2014 que mostrou um aumento no número de casos da doença, indicando o possível surgimento de um novo surto. Entre os anos de 2009 a 2014 foram notificados 821 casos de malária, causados principalmente por Plasmodium vivax (99.76\%). A doença afetou $70,15 \%$ dos homens e 29,85 das mulheres. A faixa etária mais atingida foi de 20 a 29 anos, representando $30,22 \%$ dos casos. A doença apresentou distribuição homogênea no município, ou seja, uma baixa variação nesse período. Apesar dos esforços governamentais a malária ainda é endêmica no município e um enorme desafio à saúde pública, sendo um agravo de ordem social, político e econômica, repercutindo de forma negativa na vida da população por seu caráter incapacitante.
\end{abstract}

PALAVRAS-CHAVE: Endemias no Brasil; Perfil Epidemiológico; Plasmodium.

\section{CASES OF MALARIA REGISTERED IN THE MUNICIPALITY OF CÂNDIDO MENDES, MARANHÃO, DURING THE PERIOD BETWEEN 2009 AND 2014}

\begin{abstract}
This study aimed to describe the epidemiological aspects of malaria in the municipality of Cândido Mendes, Maranhão, based on the cases reported during the period between 2009 and 2014. For this purpose, was realized a data survey from the Unified Health System (SUS) and the Information System Of Epidemiological
\end{abstract}


Surveillance for malaria (SIVEP-MALARIA), covering the period between January 2009 and December 2014. The results show the presence of malaria in the municipality, with a higher incidence in 2009 (346 cases) and a gradual reduction of cases in the following years, except in 2014, which showed an increase in the number of cases of the disease, indicating the possible new outbreak. Between 2009 and 2014, 821 cases of malaria were reported, mainly caused by Plasmodium vivax $(99.76 \%)$. The disease affected $70.15 \%$ of the men and 29.85 of the women. The age group most affected was 20 to 29 years, representing $30.22 \%$ of the cases. The disease had a homogeneous distribution in the municipality, that is, a low variation in this period. Despite the government's efforts, malaria is still endemic in the municipality and a huge public health challenge, being an aggravation of social, political and economic order, negatively affecting the life of the population due to its disabling character.

KEYWORDS: Endemic diseases in Brazil; Epidemiological Profile; Plasmodium.

\section{INTRODUÇÃO}

A malária é uma doença infecciosa causada por protozoários do gênero Plasmodium, sendo as espécies Plasmodium vivax, Plasmodium malarie e Plasmodium falciparum responsáveis pela doença no Brasil, transmitida pela picada da fêmea do mosquito do gênero Anopheles (BRAGA \& FONTES, 2004; BRASIL, 2014). A malária é uma das doenças mais prevalentes no globo terrestre, sendo considerada endêmica em 95 países e atualmente, cerca de 3,2 bilhões de pessoas estão em risco de contrair a doença (WHO, 2015). No Brasil, em 2014, foram registrados 144.111 casos de malária e 38 mortes por ocasião da doença, desse total $99,61 \%$ foram registrados na Região Amazônica sendo notificados 1.396 no Estado do Maranhão (BRASIL, 2015).

Os casos de malária registrados na região amazônica englobam os estados do Acre, Amazonas, Amapá, Maranhão, Mato Grosso, Pará, Rondônia, Roraima e Tocantins (PARISE et al., 2012; LAPOUBLE et al., 2015). Esses estados apresentam condições ecológicas propícias para o desenvolvimento e sobrevivência do vetor e, condições socioeconômicas e ambientais que favorecem a transmissão da doença (LAPOUBLE et al., 2015).

No Maranhão o maior número de registros de casos de malária se concentra na região norte do Estado, onde as condições climáticas, aspectos demográficos e a prática de desmatamento para a extração de madeira são semelhantes ao que ocorrem em outros estados amazônicos (SILVA et al., 2016). Tais fatores favorecem a reprodução do mosquito do gênero Anopheles, vetor da doença, que encontra condições ideais para seu desenvolvimento (LOPES et al., 2013; MESQUITA et al., 2013).

Historicamente o maior registro de malária no Estado do Maranhão ocorreu no ano 2000 que totalizou 78.781 casos (LOPES et al., 2013). Embora haja, atualmente, atuação de programas de controle e prevenção da malária, ela ainda é considerada um importante problema de saúde pública, principalmente por atingir a população rural e pessoas pertencentes às camadas sociais de menor nível socioeconômico (SOUSA et al., 2015).

A epidemiologia é a ciência responsável por descrever, conhecer e identificar as variáveis que compõem os processos saúde-doença, cabendo a ela, portanto, a análise dos aspectos relacionados a este processo. Quanto a isso, ressalta-se que a construção de perfis epidemiológicos é de extrema importância, visto que descrevem 
as variáveis envolvidas no processo saúde-doença, apresentando-as de forma organizada e esquematizada. A utilização dessa ferramenta é fundamental nos programas de controle e prevenção de doenças, norteiam as medidas e ações empregadas no combate e prevenção das doenças (ANDRADE et al., 2012).

O município maranhense de Cândido Mendes, assim como e em outros municípios amazônicos, tem sua atividade econômica concentrada na pesca, agricultura e extrativismo. Estas atividades rurais expõem sua população a áreas de risco de contaminação por malária (CANTANHEDE, 2006; MACIEL et al., 2013). Nos últimos anos apesar dos esforços dos programas de controle de endemias houve um aumento crescente no número de casos da doença em nível de estado, sendo preocupante uma máxima observada no ano de 2014 (BRASIL, 2014; SILVA et al., 2016).

Nesse sentido, o presente trabalho pretende descrever os aspectos epidemiológicos da malária no município de Cândido Mendes, Maranhão, baseado nos casos notificados no período de janeiro de 2009 a dezembro de 2014, com o intuito de traçar o perfil da doença em Cândido Mendes.

\section{MATERIAL E MÉTODOS}

O estudo foi realizado no município de Cândido Mendes (Figura 1) no Estado do Maranhão, localizado na chamada Amazônia Maranhense, região do Estado pertencente à Amazônia Legal. O município situa-se na mesorregião Oeste do Maranhão, microrregião do Gurupi, porção noroeste do Estado, fazendo parte das chamas Reentrâncias Maranhenses (CANTANHEDE, 2006). O município possui uma área territorial 1.640,756 km² e população estimada em 19.827 (IBGE 2016).

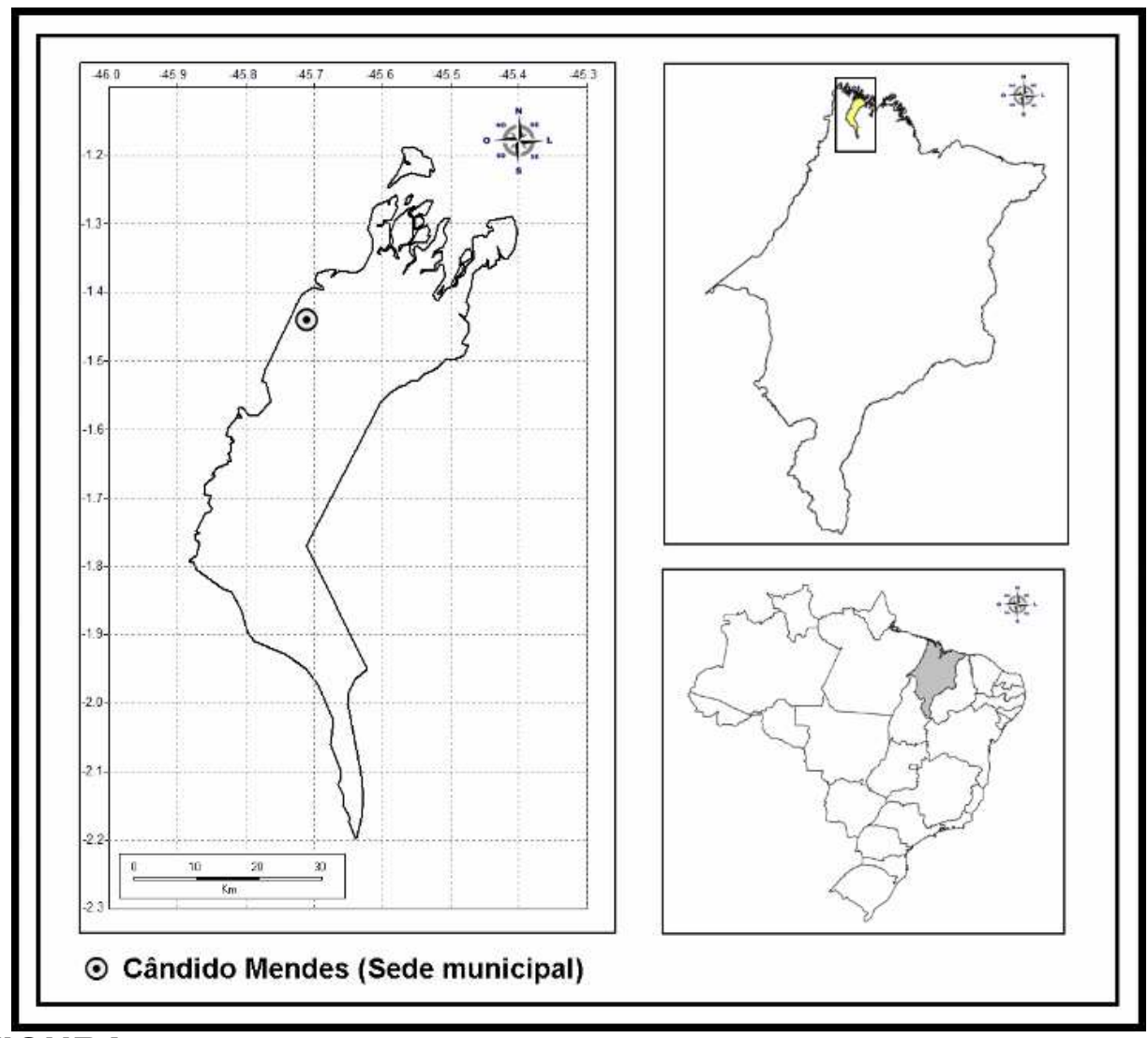

FIGURA 1. Localização do município de Cândido Mendes, Maranhão. 
Por se encontrar situado no oeste do Maranhão, o município de Cândido Mendes reflete toda uma estrutura de Floresta Amazônica. Apresenta cobertura vegetal de floresta ombrófila, floresta de dossel mais denso e fechado. Nas áreas litorâneas há presença de manguezais e no interior do mesmo, localizam-se áreas de mosaicos com florestas degradadas, restingas e transição para Matas de Cocais (CANTANHEDE, 2006).

A geomorfologia da região é constituída de um litoral com rias afogadas, convertidas em planícies aluviais. O clima é característico amazônico com uma temperatura média de $27^{\circ} \mathrm{C}$, umidade alta e precipitação média anual entre $2.400 \mathrm{e}$ $2.800 \mathrm{~mm}$ anuais, sendo considerado o município de maior índice pluviométrico em todo o Estado (CANTANHEDE, 2006)

A economia do munícipio concentra-se no setor primário dando maior ênfase a atividade pesqueira, seguida da pecuária e agricultura. O setor secundário é representado por pequenas indústrias tais como movelarias, olarias e fábricas de gelo. No setor de serviços, encontram-se estabelecimentos comerciais de pequeno e médio porte e o serviço público constitui a grande fonte de emprego e trabalho. Nota-se também no município a presença de atividade de extração mineral, muitas vezes ilegal, com abertura de garimpos a céu aberto, nas proximidades dos rios da região (CANTANHEDE, 2006; SILVA, 2017).

O presente estudo foi descritivo e retrospectivo e utilizou os dados do Sistema de Informação da Vigilância Epidemiológica (SIVEP - MALÁRIA/MA), órgão que pertence ao Ministério da Saúde, que notifica casos de malária de pacientes atendidos pela rede do Sistema Único de Saúde (SUS), em todo território nacional.

Os dados utilizados neste trabalho foram disponibilizados pela Secretaria de Saúde de Cândido Mendes e pelo Núcleo de Vigilância Epidemiológica da Fundação Nacional de Saúde (FUNASA). O material abrange todos os casos notificados como positivo para malária durante o período compreendido entre 2009 a 2014, registrados para o município de Cândido Mendes.

O diagnóstico da malária empregado foi realizado segundo regulamentação do Ministério da Saúde e utilizou o exame de gota espessa (BRASIL 2005). O presente trabalho analisou todos os casos positivos de malária, notificados e confirmados, que ocorreram no município de Cândido Mendes no período de Janeiro de 2009 a Dezembro de 2014, quando foram avaliados os seguintes aspectos epidemiológicos: número total de casos por ano, distribuição dos indivíduos infectados por sexo, idade (faixa etária) e espécie do parasita causador da doença, identificando os locais de procedência dos casos, correspondendo a uma análise quantitativa e com valores percentuais.

Os dados foram analisados descritivamente utilizando-se gráficos e tabelas, elaborados no programa Excel 2010. O mapa de distribuição de ocorrência da malária foi elaborado com base nas localidades de procedência dos casos registrados. Para isso, foram utilizados os programas computacionais de georreferenciamento Specislink e o Diva Giz 7.5. Os mapas são apresentados em sistema de coordenadas geográficas com datum WOS84 e foram elaborados pelos autores. 


\section{RESULTADOS E DISCUSSÃO}

No período de janeiro de 2009 a dezembro de 2014, o município de Cândido Mendes, foi registrada a ocorrência de malária causada pela infecção por Plasmodium vivax, Plasmodium falciparum e pela infecção mista de $P$. falciparum $e$ $P$. vivax simultaneamente. Nesse período foram notificados 821 casos positivos de malária, dos quais a prevalência de infecção se deu por Plasmodium vivax com 819 $(99,76 \%)$ dos casos registrados (Tabela 1). Ressalta-se que os casos de malária ocasionados por $P$. falciparum e pela forma mista de $P$. vivax e $P$. falciparum simultaneamente corresponderam a apenas um caso para cada agente patogênico, reforçando a incidência da doença ocasionada por $P$. vivax.

O maior número de casos de malária ocorridos em Cândido Mendes durante o período de estudo foi observado em 2009 ( $n=346)$ e o menor em $2012(n=52)$ (Tabela 1). Houve uma redução gradual do número de casos no período de estudo. Tal redução foi observada para os anos de $2010(n=121), 2011(n=113)$ e 2012 ( $n$ = 52) e possivelmente teve relação direta com a implementação e intensificação de medidas de combate e controle da doença, estabelecidas a nível estadual e municipal, que conferiram maior qualidade às políticas direcionadas a malária (construção de moradias, aquisição de viaturas, aumento no número de laboratórios) (SILVA, 2017).

Nos anos de 2013 e 2014 o município de cândido Mendes apresentou novo acréscimo no número de casos, sendo mais expressivo no ano de $2014(n=128)$, sendo estes impulsionados em decorrência da abertura de estradas vicinais que ligaram várias comunidades ao rio Maracaçumé, o que ocasionou o aumento da atividade pesqueira, maior fluxo de pessoas entre o município e municípios vizinhos que apresentavam, maior incidência da doença, possibilitando a importação da mesma para o município de Candido Mendes (SILVA, 2017).

TABELA 1: Número total de casos positivos de malária por espécie de Plasmodium, notificados no município de Cândido Mendes, Maranhão durante 2009 e 2014.

\begin{tabular}{c|c|c|c|c}
\hline \multirow{2}{*}{ Ano } & \multicolumn{3}{|c|}{ Número de Casos Positivos } & \multirow{2}{*}{ Total } \\
\cline { 2 - 5 } & Plasmodium vivax & Plasmodium falciparum & $\begin{array}{c}\boldsymbol{P} \text {. falciparum } \\
\text { P. vivax }\end{array}$ & \\
\hline 2009 & 345 & 1 & 0 & 346 \\
2010 & 121 & 0 & 0 & 121 \\
2011 & 113 & 0 & 0 & 113 \\
2012 & 52 & 0 & 0 & 52 \\
2013 & 60 & 0 & 1 & 61 \\
2014 & 128 & 0 & 0 & 128 \\
\hline Total & $\mathbf{8 1 9}$ & $\mathbf{1}$ & $\mathbf{1}$ & $\mathbf{8 2 1}$ \\
\hline
\end{tabular}

Não foram registrados casos de malária por $P$. malariae no período do estudo na região, reforçando a prevalência dos $P$. vivax e $P$. falciparum como as espécies mais 
incidentes na região. Quanto a isso é conhecida no Brasil, a transmissão da malária ocasionada por três espécies de Plasmodium: $P$. vivax, $P$. falciparum e $P$. malariae, entretanto, ressalta-se que no ano de 2011, as infecções no território brasileiro foram causadas por $P$. vivax e corresponderam a $86,61 \%$ dos casos e, a segunda espécie mais incidente foi $P$. falciparum representando $11,93 \%$ dos casos de malária registrados na Região Amazônica (BRAGA, 2004; BRASIL, 2013). No estado do Maranhão há uma tendência, de prevalência de contaminações por $P$. vivax seguido por $P$. falciparum (MESQUITA et al, 2013; LOPES et al., 2013).

No período em estudo, a relação da infecção de malária por sexo evidenciou a prevalência pelo sexo masculino com 576 casos $(70,15 \%)$, enquanto que as infecções de pessoas do sexo feminino somaram $245(29,85 \%)$. Essa prevalência foi observada em todos os anos de estudo, sendo mais evidente em 2009 (Figura 2). Esse resultado, provavelmente, deve-se ao fato de geralmente os homens trabalharem com a agricultura, pesca e extração mineral, chegando muitas vezes a manter contato com locais de criadouros do mosquito vetor da doença, sobretudo em regiões onde a malária se apresenta de forma endêmica. (MONTEIRO et al., 2013; SOUSA et al., 2015). Por outro lado, a maioria das mulheres desenvolve suas atividades diárias no ambiente doméstico ou nas proximidades de suas residências ou no peridomicílio. Entretanto é importante ressaltar que as pessoas que vivem em área endêmica de malária correm o risco de adquirir a doença em função do trabalho que o indivíduo executa e o local se a região é endêmica, devendo ainda considerar os aspectos sazonais como o período chuvoso (BARBIERI, 2005; MESQUITA et al., 2013;MACIEL et al.,2013).

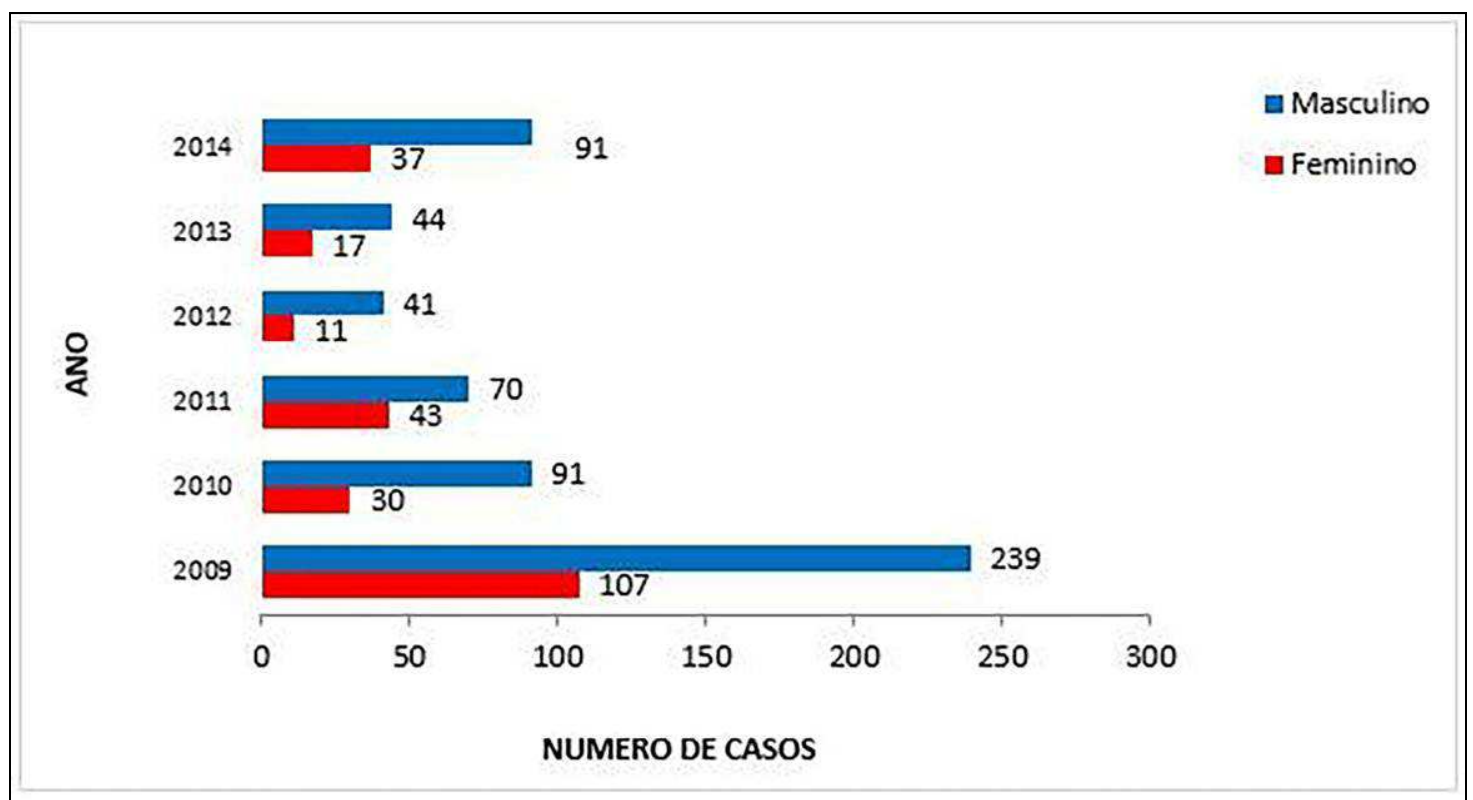

FIGURA 2. Casos positivos de malária por sexo, notificados no Munícipio de Cândido Mendes, Maranhão entre os anos de 2009 e 2014. Fonte: Os autores.

Fonte: os autores

Os casos de malária estão relacionados em sua maioria às atividades econômicas, que expõe a população as áreas de infecção. Como as atividades rurais principais se concentram na pesca, agricultura e exploração mineral a 
população masculina acaba sendo mais exposta a doença. Nesse contexto, SILVA (1989); MESQUITA et al. (2013) CATRAIO et al. (2013); MACIEL et al. (2013) reforçam a relação entre atividade exercida pelo indivíduo e o risco de contaminação.

Durante todos os anos de estudo, observou-se que a taxa de infecção de malária foi maior nas pessoas com idade no intervalo de 20 a 29 anos com 249 casos (30,22\%), seguido pela faixa dos 30 a 39 com 122 casos (14,86\%) (Figura 3). As menores taxas de infecção foram registradas na parcela mais idosa da população, sendo observados somente 2 casos no período na faixa dos 70 a 79 anos e 2 casos nos acima de 80 anos. Estes dados corroboram os encontrados por LOPES et al. (2013), que obtiveram resultado semelhante em um estudo realizado também no Estado do Maranhão. A prevalência da doença na faixa etária dita como produtiva ou economicamente ativa (PEA) indica haver uma relação entre a produção econômica e exposição a áreas de contaminação. MESQUITA et al. (2013) reforçam que no estado do Maranhão as populações rurais e semi-rurais são as mais atingidas, estando suas atividades econômicas principalmente relacionadas a lavoura, pesca, ao extrativismo vegetal e exploração mineral.

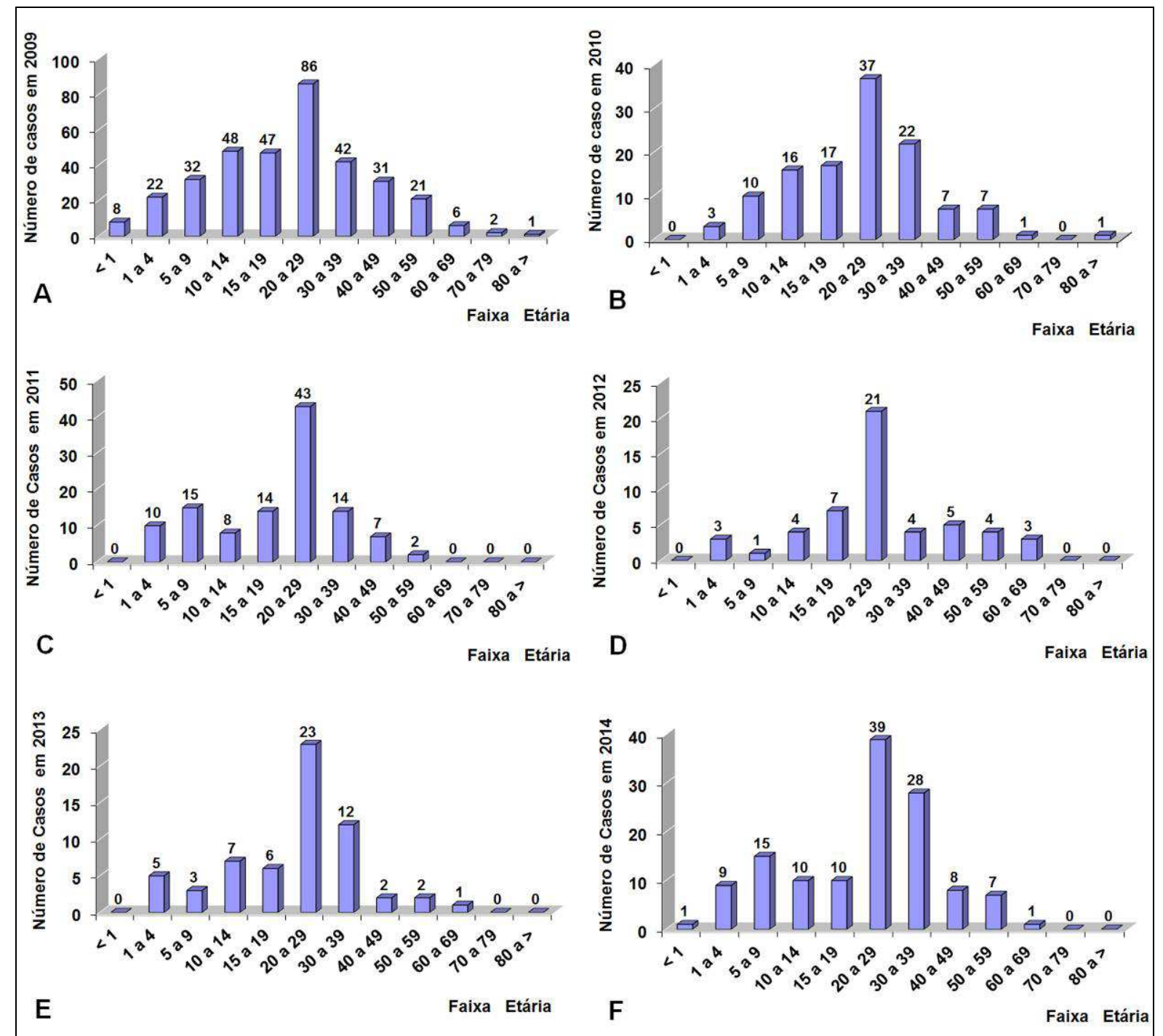

FIGURA 3. Casos positivos de malária por faixa etária, notificados no Munícipio de 
Cândido Mendes, Maranhão entre os anos de 2009 e 2014: A - 2009; B 2010; C - 2011; D - 2012; E - 20013; F - 2014.

Fonte: Os autores.

A partir dos dados disponibilizados pela Secretaria de Saúde do município de Cândido Mendes foi possível elaborar um mapa de ocorrência da malária pelo município nos anos de 2009 a 2014 (FIGURA 4). Os mapas indicam um claro padrão de distribuição homogêneo com repetição da maioria dos pontos de ocorrência, excetuando-se pequenas mudanças entre os anos estudados. Muitos autores relacionam a distribuição da malária com fatores climáticos, ambientais e sociais revelando em diversos casos um perfil irregular e heterógeno para uma mesma região tendo em vista a dinâmica desses fatores (BARBIERI, 2005; SANTOS et al., 2009; LOPES et al., 2013). Na área em questão a atividade econômica, principalmente ligada a pesca e agricultura, moradias construídas em áreas próximas a rios e corpos d'agua, presença de área de mangue e campos alagados nas proximidades da sede do município e uma estreita relação da população com o rio podem ser elencados como fatores relacionados a distribuição na área (SILVA, 2017).

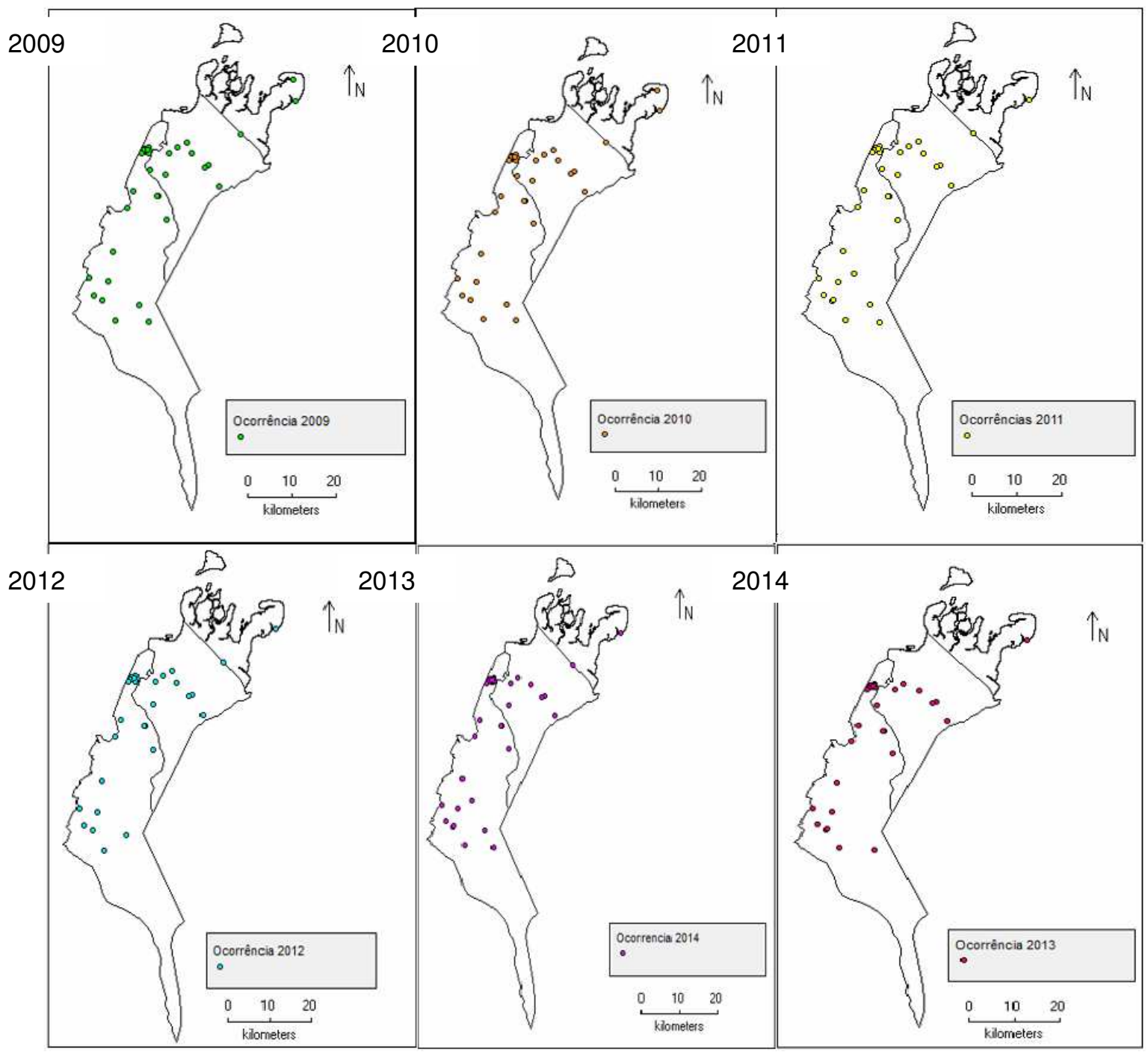

FIGURA 4. Distribuição de casos de malária notificados no Munícipio de ENCICLOPÉDIA BIOSFERA, Centro Científico Conhecer - Goiânia, v.14 n.25; p.1540 2017 
Cândido Mendes, Maranhão entre os anos de 2009 e 2014.

Fonte: Os autores.

\section{CONCLUSÃO}

Durante os anos de estudo observou-se uma redução gradativa do número de casos de malária no município de Cândido Mendes, o que foi interpretado como consequência dos esforços institucionais do local no combate e controle da doença. Entretanto, o surgimento de um acréscimo no número de casos no ano de 2014 deve ser observado com maior atenção, podendo considerar a relação desse acréscimo com a situação econômica do município que pode ter interferido na manutenção das medidas de controle.

Conclui-se ainda, que é de fundamental importância ampliar os estudos referentes a malária na região de Cândido Mendes, visto que nesta a doença ainda é endêmica, o que a torna um problema de saúde pública, nesta porção do Bioma Amazônia. Portanto, admite-se que é necessário o contínuo monitoramento da doença e também garantir subsídios para o planejamento das medidas de combate e controle da doença, melhorando a qualidade de vida da população.

\section{AGRADECIMENTOS}

Os autores agradecem a Secretaria Municipal de Saúde de Cândido Mendes e ao Núcleo de Vigilância Epidemiológica de Cândido Mendes, estado do Maranhão, pela cedência dos dados epidemiológicos da Malária no município, que foram objeto deste estudo.

\section{REFERÊNCIAS}

ANDRADE, C.T. MAGEDANZ, A. M. P. C. B. ESCOBOSA, D. M. TOMAZ, W. M. SANTINHO, C. S. LOPES, T. O. LOMBARDO, V. A importância de uma base de dados na gestão de serviços de saúde pública. Revista Einstein. 2012;10(3): 360-5.

BARBIERI, A. F. Uso de Solo e Prevalência da Malária em uma Região da Amazônia Brasileira. Caderno de Geografia, Belo Horizonte, v.15, p.9-30, 2005.

BRAGA, E. M.; FONTES C. J. F. Plasmodium - Malária. in: NEVES, D. P. Parasitologia Humana. 11 ed. São Paulo: Atheneu, 2004, p. 143-161.

BRASIL. Ministério da Saúde. Secretaria de Vigilância em Saúde. Boletim Epidemiológico, v.46, n. 25, 2015.

BRASIL. Ministério da Saúde. Secretaria de Vigilância em Saúde. Boletim Epidemiológico, v.44, n.1, 2013.

BRASIL. Ministério da Saúde. Portal da Saúde. Descrição da Doença. Brasília, 2014. Disponível em: <http://portalsaude.saude.gov.br/index.php/oministerio/principal/leia-mais-o-ministerio/662-secretaria-svs/vigilancia-de-a-az/malaria/11342-descricao-da-doenca> acesso em: 25/10/2016.

CANTANHEDE, M. V.; A constituição geográfica do município de Cândido Mendes, MA. Ed UFMA, p.138, 2006. 
CATRAIO, I. T. F. F.; LAROCCA, L. M.; MOREIRA, S. D. R.; MEDEIROS, A. R. P.; HIGAKI, N. M. A determinação social da malária: um estudo de caso na epidemiologia hospitalar. Revista uniandrade, v.12, n.2, p.125-132, 2013.

IBGE. Instituto Brasileiro de Geografia e Estatística, cidades: Maranhão, Cândido Mendes. Disponível

em: $<$ http://cidades.ibge.gov.br/xtras/perfil.php?codmun=210260> acesso em: 11/12/2016.

LAPOUBLE, O. M. M.; SANTELLI, A. C. F. S.; MUNIZ-JUNQUEIRA, M. I. Situação epidemiológica da malária na região amazônica brasileira, 2003 a 2012. Revista Panamericana de Salud Publica, v.38, n.4, p.300-306, 2015.

LOPES, N.F.S.N.; TADEI, W.P.; BRITO, L. M. O; BEZERRA, J. M. T.; PINHEIRO, V. C. S. Malária no maranhão: análise dos fatores relacionados com a transmissão no período de 2005 a 2009. Revista de Pesquisa em Saúde, v.14, n.1, p.40-44, 2013.

MACIEL, G. B. M. L.; ESPINOSA, M. M.; ATANAKA-SANTOS, M. Epidemiologia da malária no município de Colniza, Estado de Mato Grosso, Brasil: estudo descritivo do período de 2003 a 2009. Epidemiologia e Serviços de Saúde, Brasília, v.22, n.3, p.465-474, $2013 . \quad$ Disponível em <http://scielo.iec.pa.gov.br/scielo.php?script=sci_arttext\&pid=S167949742013000300011\&lng=pt\&nrm=iso $>$. acessos em 21/11/2016. http://dx.doi.org/10.5123/S1679-49742013000300011

MESQUITA, E. M.; MUNIZ, T. F.; SOUSA, A. L. S.; BRITO, C. X. L.; NUNES, S. C. M.; GRISOTTO, M. A. G. Levantamento epidemiológico da malária no estado do Maranhão, Brasil nos anos de 2007 a 2012. Revista de Ciências da Saúde, São Luís, v.15, n.1, p. 11-18, 2013.

MONTEIRO, M. R. C. C.; RIBEIRO, M. C.; FERNANDES, S. C. Aspectos clínicos e epidemiológicos da malária em um hospital universitário de Belém, Estado do Pará, Brasil. Revista Pan-Amazônica de Saúde, v.4, n.2, p.33-43, 2013. Disponível em: $<$ http://dx.doi.org/10.5123/S2176-62232013000200005

PARISE, E. V.; ARAÚJO, G. C.; CASTRO, J. G. D. Situação epidemiológica da malária no Estado do Tocantins, Brasil, a partir da emancipação política e administrativa, 1989 a 2009. Epidemiologia e Serviços de Saúde, v.21, n.1, p.129140, 2012. Disponível em: <http://dx.doi.org/10.5123/S1679-49742012000100013

SANTOS, V. R.; YOKOO, E. M.; SANTOS, R. S.; SANTOS, M. A. Fatores Socioambientais Associados a Distribuição Espacial da Malária No Assentamento Vale do Amanhecer, Município de Jurema, Estado de Mato Grosso 2005. Revista da Sociedade Brasileira de Medicina Tropical, Uberaba, v.42, n.1, 2009.

SILVA, A. R. MALÁRIA: Fotografia de uma crise no setor saúde. Editora da Universidade Federal do Maranhão, 1989. 
SILVA, A. R.; CAVALEIRO, N. N. M.; GUIMARÃES, M. C.; GONÇALVES, E. G. R. Evolução da malária no estado do maranhão: série histórica de 2009 a 2013. Revista de Patologia Tropical, v.45, n.1, p.33-41, 2016. Disponível em:<http://dx.doi.org/10.5216/rpt.v45i1.39977

SILVA, J. A. Situação epidemiológica da malária no município de Cândido Mendes-MA. Núcleo de Vigilância Epidemiológica, 03/01/2017. Entrevista concedida a Yuri Freitas da Silva.

SOUSA, J. R.; SANTOS, A. C. F.; ALMEIDA, W. S.; ALBARADO, K. V. P.; MAGNO, L. D.; ROCHA, J. A. M.; PIMENTEL, Z. N. S. Situação da malária na Região do Baixo Amazonas, Estado do Pará, Brasil, de 2009 a 2013: um enfoque epidemiológico. Revista Pan-Amazônica de Saúde. v.6, n.4, p.39-47, 2015. Disponível em: <http://dx.doi.org/10.5123/S2176-62232015000400006

WHO. Word Health Organization. Word Malaria Report 2015. Switzerland, 2015. 